\title{
Modified Dolph-Chebyshev Arrays
}

\author{
B. L. J. Rao ${ }^{1}$ \\ Radiation Laboratory, Department of Electrical Engineering, University of Michigan, \\ Ann Arbor, Mich. 48108, U.S.A. \\ (Received December 1, 1967)
}

\begin{abstract}
A method is presented to synthesize linear array patterns in which the side lobes decay very rapidly on either side of the main beam and at the same time exhibit, very closely, the optimum property of Dolph-Chebyshev patterns. When compared with Dolph-Chebyshev arrays having an equal number of elements, the side lobes of the modified arrays are considerably lower, the gain is approximately the same, and there is a small increase in the width of the main beam. For larger arrays, the increase in the beam width is negligible. In the cases where it is difficult to realize practical Dolph-Chebyshev arrays, modified arrays appear to be more practical. Some specific examples are given.
\end{abstract}

\section{Introduction}

For linear uniformly distributed arrays, the form of current distribution that has found considerable use is the Dolph-Chebyshev distribution, whose properties are optimum in the sense that it will produce the narrowest beam width for a given side-lobe level and vice versa. The resulting radiation pattern has side lobes of constant level. For some antenna applications, it may be undesirable for the side lobes to remain at a constant level as the angular separation from the main beam increases. A certain amount of decrease in the side lobes will occur depending on the directivity of the individual elements. For applications where this rate of decrease is insufficient, it is necessary to use a form of current distribution whose space factor is such that the side lobes decrease at the desired rate. Relationships for such a radiation pattern have not been worked out for the discrete-element case. Considerable work has been done on continuous distributions (Taylor, 1953, 1955). For arrays of a large number of discrete elements, the current distribution is frequently represented by a continuous function. However, where the number of elements is relatively small, say less than 20 , the continuous function representation is lacking in accuracy (Jasik, 1961), and it is desirable to represent the current distribution by a discrete number of terms. The present paper is the result of this motivation.

This paper presents a simple synthesis procedure to obtain patterns in which the side lobes decay rapidly on either side of the main beam, and at the same time exhibit, very closely, the optimum property of Dolph-Chebyshev patterns.

1 Presently assuciuted with the Research and Develuennent Directurate, Paye Cim municatiens Enpineers. Wasington. D.C. 20007.

\section{Theory}

\subsection{Even Number of Elements}

Consider an equally spaced linear broadside array of $2 \mathrm{~N}$ elements as shown in figure 1. The corresponding array factor $E_{2, v}(\psi)$ is given by

$$
E_{2 N}(\psi)=2 \sum_{k=1}^{N} I_{k} \cdot \cos (2 k-1) \frac{\psi}{2}
$$

where

$$
\begin{aligned}
\psi & =\beta d \sin \phi \\
\beta & =2 \pi / \lambda, \\
d & =\text { element spacing, } \\
\lambda & =\text { operating wavelength. }
\end{aligned}
$$

The main idea in the present approach is in recognizing the fact that,

$$
\begin{aligned}
\cos (2 k-1) \frac{\psi}{2}+\cos (2 k-3) & \frac{\psi}{2} \\
& =2 \cos \frac{\psi}{2} \cos 2(k-1) \frac{\psi}{2}
\end{aligned}
$$

and dividing each coefficient $I_{k}$ into two separate parts, and properly combining each term in (1) with the appropriate parts of adjacent terms as explained below.

Assume that,

$$
\begin{aligned}
& I_{k}=I_{k}^{\prime}+I_{k+1}^{\prime} \quad \text { for } k=1,2, \ldots, N-1, \\
& I_{V}=I_{V}^{\prime},
\end{aligned}
$$




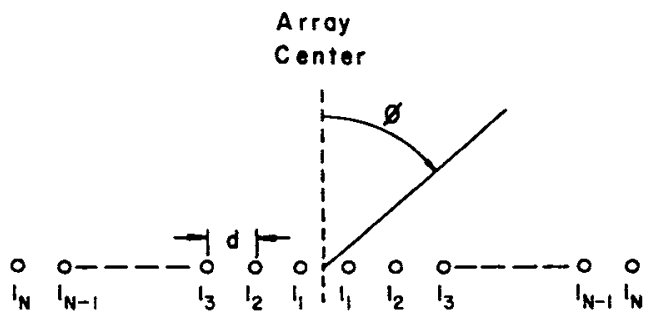

Figure 1. An equally spaced linear array of $2 \mathrm{~N}$ elements.

where the currents $I_{k}^{\prime}$ are arbitrary up to this point and to be determined later on.

Substituting the relations given by (3) in the array factor (1) and then making use of the relation (2), one can show that

$$
E_{2 . V}(\psi)=2\left[I_{1}^{\prime} \cos \frac{\psi}{2}+2 \sum_{2}^{N} I_{k}^{\prime} \cos \frac{\psi}{2} \cos 2(k-1) \frac{\psi}{2}\right] .
$$

This can be rewritten in a convenient form

$$
E_{2 . x}(\psi)=2 \cos \frac{\psi}{2} \sum_{i}^{N} A_{k}^{\prime} \cos 2(k-1) \frac{\psi}{2}
$$

where $\quad A_{k}^{\prime}=2 I_{k}^{\prime} \quad$ for $k=2,3, \ldots ., N$,

and $\quad A_{1}^{\prime}=I_{1}^{\prime}$.

Noting that the factor with summation sign in (5) is nothing but a uniform array factor of $2 N-1$ (odd number) elements, we have

$$
E_{2 . Y}(\psi)=2 \cos \frac{\psi}{2} E_{2 . Y-1}(\psi),
$$

where $\quad E_{2 . k-1}(\psi)=\sum_{1}^{x} A_{k}^{\prime} \cos 2(k-1) \frac{\psi}{2}$.

Previously the coefficients $A_{k}^{\prime}$ were arbitrary; they are now determined by applying Dolph-Chebyshev theory such that $E_{2, v-1}(\psi)$ has a Chebyshev pattern. Once the coefficients $A_{k}^{\prime}$ are determined, it is straightforward to determine the currents $I_{k}^{\prime}$ and then $I_{k}$ from the relations given by (6) and (3). Then the array factor $E_{. .1}(\psi)$ becomes

$$
E_{2 . X}(\psi)=2 \cos \frac{\psi}{2} E_{2 . V^{\prime}-1}^{d}(\psi)
$$

Note that the superscript $d$ denotes a Chebyshev array factor.

Since the function $\cos \frac{\psi}{2}$ of (8) is approximately equal to unity near the main beam, it is evident that the $3-\mathrm{dB}$ beam width, for $N$ moderately large, will correspond to the Chebyshev array factor of $2 \mathrm{~N}-1$ elements, and the side-lobe levels will be decreased below the Chebyshev level, as the angular separation from the main beam is increased. These and other pertinent points are discussed in more detail in section 2.3 .

\subsection{Odd Number of Elements}

When the array consists of an odd number of elements, the procedure is similar except for a slight modification explained below. The array factor of a uniform linear array of an odd number of elements is in general given by

$$
E_{2 N-1}(\psi)=\sum_{1}^{N} B_{k} \cos 2(k-1) \frac{\psi}{2}
$$

where the coefficients $B_{k}$ are related to the element currents $J_{k}$ as

$$
B_{k}=2 J_{k} \quad \text { for } k=2,3, \ldots, N \text {, }
$$

and $\quad B_{1}=J_{1}$.

Now assume that

$$
\begin{aligned}
& B_{1}=B_{1}^{\prime} \\
& B_{k}=B_{k-1}^{\prime}+B_{k}^{\prime} \quad \text { for } k=2,3, \ldots, N-1,
\end{aligned}
$$

and $B_{N}=B_{N-1}^{\prime}$.

Substituting the relations given by (11) in the array factor (9) and then using the relation in (2), we show that

$$
E_{2 N-1}(\psi)=2 \cos \frac{\psi}{2} \sum_{1}^{N-1} B_{k}^{\prime} \cos (2 k-1) \frac{\psi}{2} .
$$

Noting that the factor to be summed in (12) is an array factor of $2 N-2$ elements, we have

$$
E_{2 N-1}(\psi)=2 \cos \frac{\psi}{2} E_{2 N-2}(\psi)
$$

Previously the coefficients $B_{k}^{\prime}$ were arbitrary; they are now determined by applying Dolph-Chebyshev theory such that $E_{2 N-2}(\psi)$ has a Chebyshev pattern. Once the coefficients $B_{k}^{\prime}$ are found, it is straightforward to determine the necessary excitations $J_{k}$ using relations (10) and (11). The corresponding array factor becomes

$$
E_{2, N-1}(\psi)=2 \cos \frac{\psi}{2} E_{2 N-2}^{d}(\psi) .
$$

As in the case of an even number of elements, the side-lobe levels are decreased according to the factor $\cos \frac{\psi}{2}$, and the main beam width corresponds to the Chebyshev pattern of $2 N-2$ elements when the actual number of elements is $2 N-1$. 


\subsection{Specific Examples and Discussion of the Results}

Some general conclusions may be reached before discussing any specific examples. From the final array factors given by (8) and (14) for an even and odd number of elements, it is evident that any array factor could be expressed as a product of $\cos \frac{\psi}{2}$ and a Chebyshev array factor of $M-1$ elements (where $M$ is the total number of elements in the array being either an even or odd integer). If $M$ is large (say greater than 10), the effect of $\cos \frac{\psi}{2}$ on the main beam is negligible. Therefore, the 3-dB beam width corresponds to a Chebyshev array of $M-1$ elements, and is given to a good approx imation by Jasik (1961),

$$
\phi_{M-1}=\frac{A \lambda}{(M-1) d},
$$

where $A$ is a constant and depends on the required side-lobe level.

If the array elements are designed to have a Chebyshev pattern with the same side-lobe level, the corresponding 3-dB beam width will be

$$
\phi_{M}=\frac{A \lambda}{M d}
$$

The percentage of increase in beam width is then given by

$$
\frac{100}{M-1} \text { percent, }
$$

which is about 9 percent for $M$ as low as 12 , and decreases as the number of elements is increased. Actually the result given by (16) applies to larger arrays in which the first side lobe is not affected by $\cos \frac{\psi}{2}$. So the 9 percent increase for $M=12$ is on the pessimistic side, and the actual value is found when specific examples are considered. There, it is shown that the actual beam broadening is about 6 percent instead of 9 percent as predicted by the approximate formula given here.

Since the side-lobe levels are decreased below the Chebyshev side-lobe level in accordance with the term $\cos \frac{\psi}{2}$, it is convenient for later use to plot this reduction in $\mathrm{dB}$ as a function of $\phi$, as given in figure 2 for the case of $d=\lambda / 2$. From this it is evident that the effect of $\cos \frac{\psi}{2}$ is negligible for $\phi<10^{\circ}$. The side lobes are reduced by $3 \mathrm{~dB}$ at $\phi=30^{\circ}$ and $13.6 \mathrm{~dB}$ at $\phi=60^{\circ}$. Hence, the far out side lobes are reduced much more than the side lobes near the main beam.
To study the merits and demerits of the modified Chebyshev arrays as compared to Chebyshev arrays, two specific designs having 12 and 20 elements, with interelement spacing $d=\lambda / 2$, are considered using the theory developed here. The basic design is for sidelobe levels of 20,25 , and $30 \mathrm{~dB}$ corresponding to the Chebyshev pattern term. The actual levels of first and second side lobes are determined by taking into consideration the effect of the $\cos \frac{\psi}{2}$ term using figure 2 and the positions of the side lobes. The results are given in table 1. The first and second side lobes of the 20 -element arrays are reduced only $0.3 \mathrm{~dB}$ and $0.7 \mathrm{~dB}$, respectively, whereas the first and second side lobes of 12-element arrays are reduced approximately $1 \mathrm{~dB}$ and $2.5 \mathrm{~dB}$, respectively. These results are used later to compare the characteristics of the arrays designed here with the Dolph-Chebyshev arrays with the same number of elements and side-lobe levels.

The current distribution of the modified arrays and the actual Chebyshev arrays with the same sidelobe level (rounded off to the nearest $\mathrm{dB}$ ), is given in table 2, for the case of 20 elements. The values for Chebyshev arrays are obtained from the available tables (Brown and Scharp, 1958). As may be noted from this table, in some cases the ratio of maximumto-minimum excitation is larger for the modified arrays. However, in the case of Dolph-Chebyshev arrays having a large number of elements, the current in the end elements can be considerably higher than in the adjacent elements (as can be noted from table 2 for the 20-dB case). Feeding the end elements in this manner is a difficult problem, particularly for waveguide slot arrays. In such cases the current distribution of the modified arrays may be more practical with a smaller ratio of maximum-to-minimum excitation (see the $20-\mathrm{dB}$ case in table 2), due to the fact that the current in the end element is always less than the adjacent element.

The directivity and beam-width characteristics are compared in table 3 . The values for the Chebyshev arrays are obtained by interpolation from available tables (Brown and Scharp, 1958). Since the effect of $\cos \frac{\psi}{2}$ on the main beam is negligible, the beam widths of the modified arrays correspond to the Chebyshev array term. The results from table 3 suggest that the directivities of the modified arrays are approximately the same as the corresponding Chebyshev arrays. For the examples considered here, the maximum beam broadening for the modified arrays is about 6 percent. For 20-element arrays, the beam broadening is much less.

\subsection{Extension To Further Reduce the Side-Lobe Levels}

The reduction in side-lobe levels due to the term $\cos \frac{\psi}{2}$ should be sufficient for most practical cases. However, in those designs where this reduction is 


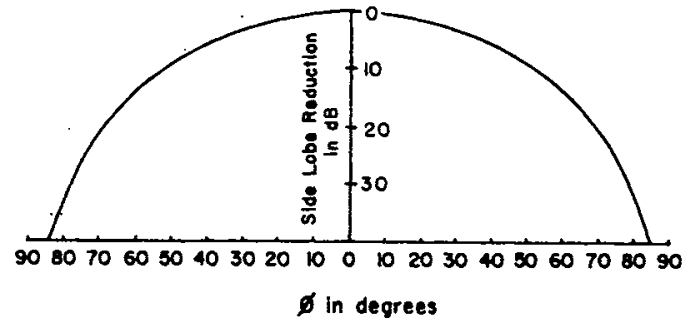

FIGURE 2. Side-lobe reduction due to the term $\cos \frac{\psi}{2}$ as a function of angular separation from the main beam $\left(\right.$ for $\left.\mathrm{d}=\frac{\lambda}{2}\right)$.

TABLE 1. First and second side-lobe levels of modified Chebyshev arrays

\begin{tabular}{c|c|c|c|c|c}
\hline $\begin{array}{c}\text { Total } \\
\text { number of } \\
\text { elements }\end{array}$ & $\begin{array}{c}\text { Design } \\
\text { side-lobe } \\
\text { level in dB }\end{array}$ & $\begin{array}{c}\text { Position } \\
\text { of first } \\
\text { side lobe } \\
\text { (degrees) }\end{array}$ & $\begin{array}{c}\text { Actual } \\
\text { level of } \\
\text { first side } \\
\text { lobe in dB }\end{array}$ & $\begin{array}{c}\text { Position } \\
\text { of second } \\
\text { side lobe } \\
\text { (degrees) }\end{array}$ & $\begin{array}{c}\text { Actual } \\
\text { level of } \\
\text { second } \\
\text { side lobe } \\
\text { in dB }\end{array}$ \\
\hline & & & & & \\
20 & 30 & 10.5 & 30.4 & 15.3 & 30.8 \\
20 & 25 & 9.7 & 25.3 & 14.8 & 25.7 \\
20 & 20 & 8.8 & 20.3 & 14.2 & 20.7 \\
12 & 30 & 18.8 & 31.1 & 27.8 & 32.6 \\
12 & 25 & 17.4 & 26.0 & 26.9 & 27.4 \\
12 & 20 & 15.8 & 20.8 & 25.9 & 22.2 \\
\hline
\end{tabular}

TABLE 2. Comparison of current distributions of arrays with 20 elements and half-wavelength spacing

\begin{tabular}{|c|c|c|c|c|c|c|}
\hline \multirow{2}{*}{$\begin{array}{l}\text { Ele- } \\
\text { ment } \\
\text { cur- } \\
\text { rents }\end{array}$} & \multicolumn{3}{|c|}{ Modified Chebyshev arrays } & \multicolumn{3}{|c|}{ Dolph-Chebyshev arrays } \\
\hline & $\begin{array}{l}\text { First side- } \\
\text { lobe level } \\
30.4 \mathrm{~dB}\end{array}$ & $\begin{array}{c}\text { First side- } \\
\text { lobe level } \\
25.3 \mathrm{~dB}\end{array}$ & $\begin{array}{l}\text { First side. } \\
\text { lobe level } \\
20.3 \mathrm{~dB}\end{array}$ & $\begin{array}{c}\text { Side-lobe } \\
\text { level } \\
30 \mathrm{~dB}\end{array}$ & $\begin{array}{c}\text { Side-lobe } \\
\text { level } \\
25 \mathrm{~dB}\end{array}$ & $\begin{array}{c}\text { Side-lobe } \\
\text { level } \\
20 \mathrm{~dB}\end{array}$ \\
\hline $\begin{array}{l}I_{1} \\
I_{2} \\
l_{5} \\
I_{4} \\
I_{3} \\
I_{6} \\
I_{7} \\
I_{8} \\
I_{5} \\
I_{10}\end{array}$ & $\begin{array}{l}1.0 \\
.967 \\
.904 \\
.815 \\
.707 \\
.588 \\
.467 \\
.350 \\
.306 \\
.159\end{array}$ & $\begin{array}{l}1.0 \\
.973 \\
.921 \\
.848 \\
.756 \\
.653 \\
.543 \\
.433 \\
.464 \\
.275\end{array}$ & $\begin{array}{l}1.0 \\
.979 \\
.939 \\
.881 \\
.808 \\
.723 \\
.630 \\
.533 \\
.737 \\
.495\end{array}$ & $\begin{array}{l}1.0 \\
.970 \\
.912 \\
.831 \\
.732 \\
.620 \\
.505 \\
.391 \\
.286 \\
.326\end{array}$ & $\begin{array}{l}1.0 \\
.976 \\
.929 \\
.862 \\
.778 \\
.682 \\
.579 \\
.474 \\
.371 \\
.567\end{array}$ & $\begin{array}{l}1.0 \\
.982 \\
.945 \\
.893 \\
.826 \\
.748 \\
.662 \\
.570 \\
.477 \\
1.028\end{array}$ \\
\hline
\end{tabular}

TABLE 3. Directivity and 3-dB beam width comparison ,

\begin{tabular}{c|c|c|c|c|c}
\hline \multirow{2}{*}{$\begin{array}{c}\text { Number of elements } \\
\text { in the array }\end{array}$} & \multirow{2}{*}{$\begin{array}{c}\text { First side- } \\
\text { lobe level } \\
\text { in dB }\end{array}$} & \multicolumn{2}{|c|}{$\begin{array}{c}\text { Modified Chebyshev } \\
\text { arrays }\end{array}$} & \multicolumn{2}{|c}{$\begin{array}{c}\text { Chebyshev arrays with } \\
\text { same sidelobe level }\end{array}$} \\
\cline { 3 - 6 } & $\begin{array}{c}\text { Beam width } \\
\text { (degrees) }\end{array}$ & Directivity & $\begin{array}{c}\text { Beam width } \\
\text { (degrees) }\end{array}$ & Directivity \\
\hline 20 & 30.4 & 6.7 & 12.21 & 6.34 & 12.36 \\
20 & 25.3 & 6.2 & 12.51 & 5.93 & 12.67 \\
20 & 20.3 & 5.7 & 12.8 & 5.43 & 12.8 \\
12 & 32.6 & 11.8 & 9.8 & 11.1 & 9.9 \\
12 & 27.4 & 11.0 & 10.1 & 10.4 & 10.3 \\
12 & 22.2 & 10.1 & 10.4 & 9.5 & 10.5 \\
\hline
\end{tabular}

insufficient, the far out side lobes could be further reduced by combining the methods given in sections 2.1 and 2.2. From (7) and (13), it is noted that the array factor of $M$ ( $M$ could be either even or odd) elements can be expressed as

$E_{M}(\psi)=2 \cos \frac{\psi}{2} E_{M-1}(\psi)=2^{2} \cos ^{2}\left(\frac{\psi}{2}\right) E_{M-2}(\psi)=\ldots$

In general, one could express

$$
E_{M}(\psi)=2^{k} \cos ^{k}\left(\frac{\psi}{2}\right) E_{M-k}(\psi)
$$

and then impose the condition that $E_{M-k}(\psi)$ should have a Chebyshev pattern.

By choosing the proper value for $k$, one may obtain a sufficient reduction in the side lobes at the expense of increased beam width and the ratio of maximum-tominimum excitation.

As a matter of academic interest, consider the special case of $k=M$. For this, the array factor reduces to

$$
E_{M}(\psi)=2^{M} \cos ^{M}\left(\frac{\psi}{2}\right)
$$

The pattern factor given by (20) corresponds to a binomial current distribution. The current distribution obtained by the methods used here agree exactly with the binomial distribution.

\section{Conclusion}

In this paper, a method is presented to synthesize linear array patterns in which the side lobes decay rapidly on either side of the main beam and at the same time exhibit, very closely, the optimum property of Dolph-Chebyshev patterns. It is found that, in some cases, the ratio of maximum-to-minimum excitation is larger for the modified arrays when compared to Chebyshev arrays having the same number of elements and first side-lobe levels. However, in the case of Dolph-Chebyshev arrays employing a large number elements, the current in the end elements can be considerably higher than in the adjacent elements. Since this is difficult to realize in practice, the modified arrays are more practical because in those cases the ratio of maximum-to-minimum excitation is smaller and the currents in the end elements are always less than that of the adjacent elements.

For the examples considered, the modified arrays, when compared to Chebyshev arrays, have approximately the same directivities. The maximum beam broadening is about 6 percent for 12 element arrays and 5 percent for 20 element arrays.

The author expresses his deep appreciation to R. E. Hiatt, J. E. Ferris, and D. L. Sengupta for reading the 
manuscript and making several valuable suggestions to improve it.

\section{References}

Brown, L. B., and G. A. Scharp (1958), Tschebyscheff antenna distribution, beamwidth, and gain tables, Naval Ordnance Lab., Corona, Calif., NOLC Rept. No. 383.

Jasik, H, ed. (1961), Antenna Engineering Handbook, 1st ed., 2-202-24 (McGraw-Hill Book Co., Inc., New York, N.Y.).
Taylor, T. T. (1953), One parameter family of line sources producing modified $\sin \pi u / \pi u$ patterns, Hughes Aircraft Co., Tech. Memo. 324, Culver City, Calif.

Taylor, T. T. (1955), Design of line-source antennas for narrow beamwidth and low sidelobes, IRE Trans. Ant. Prop., AP-3, No. 1, 16-28.

(Paper 3-5-383) 